\title{
CI Calculations of the Ground State Ionization Potential and Electron Affinity of Aluminum
}

\author{
ADNAN YOUSIF HUSSEIN* \\ Department of Physics, College of Education, University of Mustansiriya, Baghdad, Iraq \\ (Received May 2, 2015; in final form May 12, 2015)
}

\begin{abstract}
Nonrelativistic configuration interaction study for $\mathrm{Al}^{+}, \mathrm{Al}$ and $\mathrm{Al}^{-}$are presented, included calculations of ionization potential and electron affinity of the ${ }^{2} P^{o}$ ground state of Al. CI calculations up to double, triple and quadrupole excitations for $\mathrm{Al}^{+}, \mathrm{Al}$ and $\mathrm{Al}^{-}$, respectively, where neon fixed core is considered. Appropriate Slater type basis functions were developed suitable to recover both of core-valence and core-core correlation effect. The relativistic effect on both ionization potential and electron affinity are taken into account at the relativistic Hartree-Fock level. The calculated electron affinity is $432.811 \mathrm{meV}$ which is in excellent agreement with experimental value of Sheer et al. $432.83(5) \mathrm{meV}$, whereas the calculated ionization potential is $5985.764 \mathrm{meV}$, the latter is in perfect agreement with experimental value of $5985.768 \mathrm{meV}$.
\end{abstract}

DOI: 10.12693 /APhysPolA.128.268

PACS: $31.15 . \mathrm{V}-$

\section{Introduction}

During the past years, many calculations of electron affinity (EA) were carried out on the ground state ${ }^{2} P^{o}$ of $\mathrm{Al}$ with respect to ground state ${ }^{3} \mathrm{P}$ of $\mathrm{Al}$, these calculations were started in 1992 with Arnau et al. [1] where they applied configuration interaction (CI) with pseudopotentials and obtained an EA of $450 \mathrm{meV}$.

Dunning and co-workers $[2,3]$ have developed families of Gaussian basis sets optimized for correlated calculations on the valence electrons of atoms and molecules, these basis sets later used to calculate electron affinity with multireference single and double excitation configuration interaction (MRSD-CI) with the augmented correlation consistent polarized valence basis set (augcc-pvDZ) and reported an EA of $437 \mathrm{meV}$ [4]. Eliav et al. applied relativistic coupled-cluster (RCC) method in a large four-component Gaussian-spinor basis to calculate electron affinities as well as ionization potential (IP) of group-13 elements including $\mathrm{Al}$ and reported an EA of $427 \mathrm{meV}$ and IP of $5991 \mathrm{meV}$ [5]. In the same year, multiconfiguration Dirac-Fock (MCDF) method due to Wijesundera [6] were used through out to obtain an EA of $433 \mathrm{meV}$. Benchmark $a b$ initio and density functional calculations by de Oliveira et al. [7] has yielded an EA, which agrees with the last experimental value of Scheer et al. [8]. The important feature of de Oliveira calculations is the inclusion of inner-shell correlation (corevalence interaction) which lead to crucial contribution to EA.

The present work introduces a suitably developed oneelectron basis set of the Slater type orbital (STO) to treat both core-valence and core-core correlation effects. These basis set are equivalent to Gaussian basis set of

*e-mail: adnanyosif@yahoo.com
Dunning and co-workers (correlation consistent polarized core-valence cc-pCVXZ) [9]. These basis set are used to calculate accurate electron affinity and ionization potential of the ground state of aluminum in the frame work of nonrelativistic CI method.

CI calculations up to triple and quadrupole excitations are limited to valence correlation calculation where the negative ion $\mathrm{Al}$ and the neutral atom $\mathrm{Al}$ are treated as four- and three-electron system, respectively. The relativistic correction is added at the Dirac-Fock level by making relativistic Hartree-Fock calculations on the related levels ${ }^{1} S,{ }^{2} P_{1 / 2}$, and ${ }^{3} P$. In Sect. 2, we describe the $\mathrm{CI}$ theory employed to calculate ionization potential and electron affinity and where we briefly review the construction of the CI wave function. The orbital basis set used are taken up in Sect. 3. In Sect. 4, the approach of the approximate full configuration interaction (FCI) in the frame work of a priori selected configuration interaction (SCI) with its corresponding truncation energy error [10] and CI by parts CIBP [11] are presented. Results and discussion is presented in Sect. 5 and finally conclusions are drawn in Sect. 6.

\section{CI theory}

The matrix form of the Schrödinger equation is

$$
\boldsymbol{H} \boldsymbol{C}_{\mu}=E_{\mu}^{\mathrm{FCI}} \boldsymbol{C}_{\mu},
$$

where $E_{\mu}^{\mathrm{FCI}}$ is the full CI energy which is an upper bound energy of the system under consideration, $\boldsymbol{C}_{\mu}$ is column vector of the CI coefficients, and $\boldsymbol{H}$ is the matrix element representation in terms of the Slater determinants or $N$ electron symmetry eigenfunctions constructed from given orbital basis.

In CI nomenclature, Eq. (1) is called full CI (FCI) equation [12].

The exact eigenvalue $E_{\mu}$ of the Schrödinger equation can be expressed as

$$
E_{\mu}=E_{\mu}^{\mathrm{FCI}}+\Delta E_{\mu}^{\mathrm{OBI}}
$$

where $\Delta E_{\mu}^{\mathrm{OBI}}$ is the basis set incompleteness error [13]. 
In CI approach the many electron wave function is expanded as linear combination of configuration state functions $F_{g K}[14]$ :

$$
\Psi=\sum_{K=1}^{K_{x}} \sum_{g=1}^{g_{K}} F_{g K} C_{g K} .
$$

The configuration state function $F_{g K}$ are obtained as successively orthogonalized symmetric projections of Slater determinants, they may be expressed as linear combination of $n_{k}$ Slater determinants $D_{i K}$ :

$$
F_{g K}=O\left(L^{2}, S^{2}\right) \sum_{i=1}^{g} D_{i K} b_{i}^{g}=\sum_{i=1}^{n_{K}} D_{i K} c_{i}^{g},
$$

where $O\left(L^{2}, S^{2}\right)$ are idempotent projection operators for $L^{2}$ and $S^{2}$. $K$ and $g$ labels for configuration and degenerate element, respectively.

Triply and higher excited configurations are classified into disconnected and connected ones. Disconnected configurations are those that can be expressed as products of combinations of lower excited ones, whereas connected configurations are all others [10].

In general, the set of all excited configurations, $F_{g K}$ are divided into classes and in turn all configurations differing just in the labels of the virtual orbital radial functions are called subclass, where the coefficients $b_{i}^{g}$ and $c_{i}^{g}$ in Eq. (4) are the same for different configurations within a given subclass.

\section{Basis set construction}

In our calculations, the radial part $R_{i l}$ of the orbital is expressed as linear combination of normalized Slater type orbital STOs $S_{j l}$ :

$$
R_{i l}=\sum_{j} S_{j l} a_{j l i}
$$

where

$$
\begin{aligned}
& \left.S_{j l}=N_{j l} r^{(} n_{j}-1\right) \exp \left(-Z_{j l} r\right), \\
& \left.N_{j l}=\left(2 Z_{j l}\right)^{(} n_{j}+1 / 2\right)\left[\left(2 n_{j}\right) !\right]^{-1 / 2} .
\end{aligned}
$$

The representation of orbitals in terms of STOs for valence $\mathrm{CI}$ calculations of $\mathrm{Al}$ and $\mathrm{Al}^{+}$are developed from initial STOs of the Hartree-Fock [15] quality of $9 s$ and $8 \mathrm{P}$, and an additional reoptimized functions of $3 d$ and $2 f$ were considered to describe the diffuse charge distribution of $\mathrm{Al}^{-}$. Thereafter, the correlation orbital space wave function is expanded by inclusion all singles and doubles excitations outside the neon fixed core up to orbital harmonic $l=20$, leading to 121, 168 and 186 energy optimized STOs for ${ }^{1} S,{ }^{2} P^{o}$ and ${ }^{3} P$ states respectively, where it is important to maintain the initial $9 s$ and $8 \mathrm{P}$ fixed during the optimization process. Later the resulting final basis set are used to make the valence correlation full CI calculations for $\mathrm{Al}$ and $\mathrm{Al}^{-}$as will be discussed in the next section.

For valence-core and core-core CI calculations, the same initial STOs are used as that for valence calculations except that all pure single and double excited configurations outside neon fixed core are excluded from the configuration list, thus only pure single and double excited configurations coming from the core as well those configurations coming from mixed double excitations from neon core and valence electrons outside the core are included in the CI expansion.

In our work both core-valence $(\mathrm{CV})$ configuration interaction calculation and core-core (CC) configuration interaction calculation are done together and we denoted them by CVCC, work is in progress to separate both components.

\section{CI techniques}

Two CI techniques were performed throughout this work, the first one is the priori SCI to approximate full CI which where done by the program AUTOCL [14]. The new version of the mentioned program consists more compact and efficient energy selection thresholds for selection of subclasses and configurations.

The selection of disconnected configurations are based on the Brown formula [16]:

$$
\Delta E_{K}=\left(E-H_{K K}\right) B_{K}^{2} /\left(1-B_{K}^{2}\right),
$$

where $E$ is any pertinent energy, $H_{K} K$ is the diagonal matrix element which can be well approximated by the expectation value of any determinant belonging to the configuration $K$, and $B_{K}$ is an approximation to the configuration coefficient associated with triple and higher excited disconnected configurations [10]. The selection of $q$-excited connected configurations are based on modified Brown formula [17]:

$$
\Delta E_{K}^{\mathrm{pgy}}=\left(E-H_{K K}\right) \prod_{i=1}^{q} n_{K_{i}},
$$

where $n_{K_{i}}$ are the occupation numbers of the natural orbital which are implemented to approximate the correlation orbitals.

All triply and higher disconnected subclasses with energy contributions lesser than an energy threshold

$T_{\text {subc }}^{\text {egy }}$ are discarded and accumulated into the total truncation energy error $\Delta E^{\text {egy }}$, while the triply and higher connected subclasses with an energy contribution lesser than an energy threshold $T_{\text {subc }}^{\text {pgy }}$ are discarded and accumulated into the total truncation energy error $\Delta E^{\text {pgy }}$ such that

$$
\Delta E^{\text {egy }}=\sum_{\text {deleted subc }} \Delta E_{\mathrm{subc}}^{\mathrm{dis}}
$$

and

$$
\Delta E^{\mathrm{pgy}}=\sum_{\text {deleted subc }} \Delta E_{\mathrm{subc}}^{\mathrm{con}}
$$

where

$$
\Delta E_{\mathrm{subc}}^{\mathrm{dis}}=\sum_{K} \Delta E_{K}
$$

and

$$
\Delta E_{\mathrm{subc}}^{\mathrm{con}}=\sum_{K} \Delta E_{K}^{\mathrm{pgy}} .
$$

Selection of subclasses reduce the FCI size of energy $E^{\mathrm{FCI}}$ into a condensed space (model space) $M$ of energy $E_{M}$ 
and truncation energy errors of $\Delta E_{M}^{\mathrm{egy}}$ and $\Delta E_{M}^{\mathrm{pgy}}$ associated with that model space

$$
E^{\mathrm{FCI}}=E_{M}+\Delta E_{M}^{\mathrm{egy}}+\Delta E_{M}^{\mathrm{pgy}} .
$$

Because the size of model space is still too high to solve its corresponding eigenvalue problem, therefore another selection process is made on individual configurations. The program ATMOL is used to perform another selection with different values of energy and pseudo energy selection thresholds for each excitation of disconnected and connected configurations respectively to reduce the size of the model space to more condensed space $S$ :

$$
E_{M}=E_{S}+\Delta E_{S}^{\mathrm{egy}}+\Delta E_{S}^{\mathrm{pgy}}
$$

where the meanings of $\Delta E_{S}^{\text {egy }}$ and

$\Delta E_{S}^{\mathrm{pgy}}$ are identical as those in Eq. (14), except that the sums in Eqs. (10) and (11) are run over deleted configuration rather than subclasses.

The next step taken by the program ATMOL is the CI by part CIBP, where the $S$-space is partitioned into several subspaces $S_{0}, S_{1}, S_{2}, \ldots, S_{r}$ of dimensions $d_{0}, d_{1}, d_{2}, \ldots, d_{r}$, respectively, where $S_{0}$ is the reference space in which all CI coefficients are always variational, and all other subspaces $S_{i}, i=1,2, \ldots, r$ will be taken up variationally one after the other [18].

The final eigenvalue will satisfy

$$
E_{S}=E_{\mathrm{CIBP}}+\Delta E_{\mathrm{CIBP}} \text {, }
$$

where $\Delta E_{\mathrm{CIBP}}$ is the error due to CIBP method which can be determined through sensitivity analysis, where $\Delta E_{\mathrm{CIBP}}$ is necessarily a positive quantity. In order to make $\Delta E_{\mathrm{CIBP}}$ rather small, the energy corresponding to $S_{0}+S_{1}$ must be as close as possible to $E_{\mathrm{CIBP}}$, thus pushing up values of $d_{0}+d_{1}$ that require solving the eigenvalue problem outside RAM. After $S_{1}$ is processed, however, the remaining subspaces $S_{i}$ ( $i$ greater than 1$)$ are (automatically) chosen so that all matrix elements in $d_{0}+(i-1)+d_{i}$ fit in RAM [19].

\section{Results and discussion}

In this work, nonrelativistic CI calculations of ionization potential and electron affinity are divided into two components, the first one is the valence correlation component and the second one is CVCC correlation component. The first component included configuration interaction calculations of IP and EA at neon fixed core approximation, where only two, three and four electrons correlation for $\mathrm{Al}^{+1} S, \mathrm{Al}^{2} P^{o}$ and $\mathrm{Al}^{-}{ }^{3} P$ states respectively are considered. In this stage of calculation, excitations up to double, triple and quadrupole for ${ }^{1} S,{ }^{2} P^{o}$ and ${ }^{3} P$ states, respectively.

Table I shows angular momentum-energy convergence at singles and doubles excitations level (SD) up to harmonic $l=20$ for ${ }^{1} S,{ }^{2} P^{o}$ and ${ }^{3} P$ states for valence correlation $\mathrm{CI}$ calculations. The pattern of ${ }^{3} P$ state shows the slowest energy convergence with respect to both ${ }^{1} S$ and ${ }^{2} P^{o}$ states, and the ${ }^{1} S$ state show the fast energy convergence pattern.

TABLE I

Angular momentum-energy convergence of $\mathrm{Al}^{+}{ }^{1} S, \mathrm{Al}^{-2} P^{o}$ and $\mathrm{Al}^{-3} P$ states for CISD valence calculations. Total energies $E$ (in $\mathrm{Ha}$ ) and energy-convergence $\Delta E$ (in $\mu \mathrm{Ha}$ ).

\begin{tabular}{c|c|c|c|c|c|c}
\hline \hline$l_{\max }$ & $E\left(\mathrm{Al}^{+1} S\right)$ & $\Delta E$ & $E\left(\mathrm{Al}^{-2} P^{o}\right)$ & $\Delta E$ & $E\left(\mathrm{Al}^{-3} P\right)$ & $\Delta E$ \\
\hline 1 & -241.7133874116 & & -241.9076962793 & & -241.9104554047 & \\
2 & -241.7147899470 & 1402.535 & -241.9305783917 & 22882.112 & -241.9420820658 & 31626.661 \\
3 & -241.7150567791 & 266.832 & -241.9321116772 & 1533.285 & -241.9450824114 & 3000.346 \\
4 & -241.7151476805 & 90.901 & -241.9324624924 & 350.815 & -241.9456759472 & 593.536 \\
5 & -241.7151869539 & 39.273 & -241.9325898086 & 127.316 & -241.9458761422 & 200.195 \\
6 & -241.7152065365 & 19.583 & -241.9326473919 & 57.583 & -241.9459630522 & 86.910 \\
7 & -241.7152173957 & 10.859 & -241.9326773309 & 29.939 & -241.9460070367 & 43.984 \\
8 & -241.7152238555 & 6.460 & -241.9326944340 & 17.103 & -241.9460317353 & 24.699 \\
9 & -241.7152278625 & 4.007 & -241.9327048108 & 10.377 & -241.9460465298 & 14.794 \\
10 & -241.7152304160 & 2.553 & -241.9327115109 & 6.700 & -241.9460559750 & 9.445 \\
11 & -241.7152321731 & 1.757 & -241.9327159622 & 4.451 & -241.9460622170 & 6.242 \\
12 & -241.7152333427 & 1.170 & -241.9327190445 & 3.082 & -241.9460664110 & 4.194 \\
13 & -241.7152341862 & 0.843 & -241.9327212187 & 2.174 & -241.9460693805 & 2.969 \\
14 & -241.7152347502 & 0.564 & -241.9327227744 & 1.556 & -241.9460715388 & 2.158 \\
15 & -241.7152351748 & 0.425 & -241.9327239259 & 1.151 & -241.9460731010 & 1.562 \\
16 & -241.7152354393 & 0.264 & -241.9327247497 & 0.824 & -241.9460742689 & 1.168 \\
17 & -241.7152356399 & 0.201 & 241.9327253752 & 0.625 & -241.9460751123 & 0.843 \\
18 & -241.7152357976 & 0.158 & -241.9327258109 & 0.436 & -241.9460757466 & 0.634 \\
19 & -241.7152358748 & 0.077 & -241.9327261529 & 0.342 & -241.9460761959 & 0.449 \\
20 & -241.7152359358 & 0.061 & -241.9327263655 & 0.213 & -241.9460765519 & 0.356
\end{tabular}


The composition of the optimized orbital basis set at CISD valence correlation calculation are listed in Table II. The total energies of CISD calculated at valence correlation stage was extrapolated as a function of angular momentum up to $l=500$, using the Schwartz patterns [20] $a(l+1 / 2)^{-4}$ leading to deduce the basis set incompleteness error $\Delta E_{\mu}^{\mathrm{OBI}}$ as shown in Table II.

TABLE II

Composition of orbital basis sets for $\mathrm{Al}^{+}{ }^{1} S, \mathrm{Al}^{2} P^{o}$ and $\mathrm{Al}^{-3} P$ states and corresponding basis set incompleteness error $\Delta E^{\mathrm{OBI}}$ (in $\mathrm{Ha}$ ) for CISD valence calculations.

\begin{tabular}{|c|c|c|c|}
\hline Species & Basis set & No. of STOs & $\Delta E^{\mathrm{OBI}}$ \\
\hline $\mathrm{Al}^{+1} S$ & $\begin{array}{l}\text { 13s14p11d8f8g8h7i7k } \\
716 \mathrm{~m} 5 \mathrm{n} 5 \mathrm{o} 4 \mathrm{q} 4 \mathrm{r} 3 \mathrm{t} 3 \mathrm{u} 2 \mathrm{v} \\
\text { 2w2x1y1z }\end{array}$ & 121 & $-0.00012(1)$ \\
\hline $\mathrm{Al}^{2} P^{o}$ & $\begin{array}{l}\text { 15s16p15d13f12g11h9 } \\
\text { i9k917m7n7o6q6r5t5u } \\
\text { 4v4w3x3y2z }\end{array}$ & 168 & $-0.000009(1)$ \\
\hline $\mathrm{Al}^{-3} P$ & $\begin{array}{l}\text { 16s17p15d15f14g12h } \\
11 \mathrm{i} 11 \mathrm{k} 1019 \mathrm{~m} 9 \mathrm{n} 8 \mathrm{o} 6 \mathrm{q} 6 \mathrm{r} \\
6 \mathrm{t} 5 \mathrm{u} 5 \mathrm{v} 4 \mathrm{w} 4 \mathrm{x} 3 \mathrm{y} 3 \mathrm{z}\end{array}$ & 186 & $-0.000020(1)$ \\
\hline
\end{tabular}

The optimized STOs basis set at SD approximation are used to carry out the corresponding valence correlation FCI calculations, where CISDT for ${ }^{2} P^{o}$ state and CISDTQ for ${ }^{3} P$ state. The sizes of valence FCI space, the model CI space MCI, and the selected CI space SCI for ${ }^{2} P^{o}$ and ${ }^{3} P$ states for different excitations are presented in Table III. The details for $\mathrm{Al}^{+1} S$ state is not appear in Table III because its FCI calculation is restricted to CISD valence calculation. The estimation of valence FCI truncation energy errors which are based on the Brown formula and modified Brown formula do not exceed $-0.003 \mu \mathrm{Ha}$ for $\mathrm{Al}^{3} P$ state and approximately zero truncation energy error for $\mathrm{Al}^{2} P$. The error due to CI by part $\Delta E^{\mathrm{OBI}}$ are estimated according to sensitivity analysis [18], which lead to $\Delta E_{\mathrm{CIBP}} \ll 1 \mu \mathrm{Ha}$.

TABLE III

The CI size of the full CI space FCI, the model space MCI and the selected space SCI, for $\mathrm{Al}^{2} P^{o}$ and $\mathrm{Al}^{-3} P$ states for CI valence calculations.

\begin{tabular}{c|c|c|c|c}
\hline \hline Species & Excitation & FCI-size & MCI-size & SCI-size \\
\hline $\mathrm{Al}^{2} P$ & 3 & 822318 & 218751 & 214105 \\
$\mathrm{Al}^{3} P$ & 3 & 6084171 & 1351413 & 287277 \\
& 4 & 676787701 & 122116782 & 1339814
\end{tabular}

The relativistic correction contribution to both IP and EA have been estimated by carried out calculations at relativistic Hartree-Fock level [21] on the related atomic lowest levels ${ }^{1} S,{ }^{2} P_{1 / 2}$ and ${ }^{3} P_{0}$ belonging to ${ }^{1} S,{ }^{2} P$ and ${ }^{3} P$ states respectively. Our result for relativistic contribution to electron affinity is little bit below de Oliveira et al. [7] result, the reason for this difference is due to that in de Oliveira relativistic calculation just including the spin-orbit, Darwin and mass-velocity terms.
TABLE IV

Composition of orbital basis sets for $\mathrm{Al}^{+1} S, \mathrm{Al}^{2} P^{o}$ and $\mathrm{Al}^{-}{ }^{3} P$ states for core-valence and core-core (CVCC) correlation calculations.

\begin{tabular}{c|l|c}
\hline \hline Species & Basis set & No. of STOs \\
\hline $\mathrm{Al}^{+1} S$ & $16 \mathrm{~s} 16 \mathrm{p} 16 \mathrm{~d} 15 \mathrm{ff} 14 \mathrm{~g} 14 \mathrm{~h} 12 \mathrm{i} 12 \mathrm{k} 10$ & 175 \\
& $18 \mathrm{~m} 8 \mathrm{n} 6 \mathrm{q}$ q $5 \mathrm{r} 4 \mathrm{t} 4 \mathrm{u} 3 \mathrm{v} 2 \mathrm{w} 2 \mathrm{y} 1 \mathrm{y} 1 \mathrm{z}$ & \\
$\mathrm{Al}^{2} P^{o}$ & $16 \mathrm{~s} 18 \mathrm{p} 16 \mathrm{~d} 16 \mathrm{f} 14 \mathrm{~g} 14 \mathrm{~h} 13 \mathrm{i} 12 \mathrm{k} 10$ & 178 \\
& 18m8n6o5q5r4t4u3v2w2x1y1z & \\
$\mathrm{Al}^{-3} P$ & $16 \mathrm{~s} 18 \mathrm{p} 16 \mathrm{~d} 16 \mathrm{f} 15 \mathrm{~g} 14 \mathrm{~h} 12 \mathrm{i} 12 \mathrm{k} 10$ & 179 \\
& $19 \mathrm{~m} 7 \mathrm{n} 7 \mathrm{o} 5 \mathrm{q} 5 \mathrm{r} 4 \mathrm{t} 4 \mathrm{u} 3 \mathrm{v} 2 \mathrm{w} 2 \mathrm{x} 1 \mathrm{y} 1 \mathrm{z}$ &
\end{tabular}

The developed STOs for the CVCC correlation component calculations are reported explicitly in Table IV. These basis set are used to calculate the corresponding contribution component of both IP and EA. It is important to mention that the convergence of the CVCC correlation effect as a function of basis set optimization are much slower than that for the valence correlation contribution.

Numerical results of total energies contributions on different levels, HF and CISD and FCI for valence calculations component and the contribution of CVCC component and relativistic correction component and there corresponding contribution to the ionization potential are summarized in Table $\mathrm{V}$. The FCI valence contribution recovers $1.15 \%$ of the final value of the predicted IP, while the CVCC correlation reduce the predicted value of IP by $0.044 \%$. The smallest contribution comes from the relativistic effect where gives $0.011 \%$. Our predicted value of IP is exact to two decimal point with respect to experiment.

Similarly, in Table VI, we summarized different energies contributions and the corresponding contributions to electron affinity. The FCI valence contribution recovers $21.6 \%$ of the final value of the predicted EA which exceeds the corresponding contribution in IP. The CVCC correlation reduces the predicted value of $\mathrm{EA}$ by $3.4 \%$ which is also exceeding the corresponding value in IP. In EA calculation the relativistic effect reduces the predicted value of EA by $2.12 \%$, while the later effect increases the predicted value of IP by $0.01 \%$. Our CVCC contribution to $\mathrm{EA}$ is $-15 \mathrm{meV}$ which is below the corresponding de Oliveira et al. result of $-16.17 \mathrm{meV}$, this attributed to the reason, that in our calculation, both corevalence and core-core correlations are taken together into consideration, while de Oliveira et al. calculations are restricted to the effect of inner shell correlation which was determined as the difference between valence only and all electron $\operatorname{CCSD}(\mathrm{T})$ (coupled cluster with all single and double excitations and triple excitations) calculations using the Martin-Taylor family of core-correlation basis sets [7].

As seen from Tables $\mathrm{V}$ and VI, it is clear that CVCC component is of negative sign (reduces the ionization potential and electron affinity). Also, it should be noted that the effect of inclusion of CVCC correlation on 
both ionization potential and electron affinity are crucial to converge with the last measured EA value by Scheer et al. [8] and the experimental value of ionization potential [22]. However, the CVCC can be positive or negative. Finally, we compare our result with some previous results of theoretical ionization potential and electron affinity calculations as shown in Table VII.

TABLE V

Total energies (in Ha) on different levels of valence calculation, core-valence and core-core (CVCC) and relativistic effect (rel) for $\mathrm{Al}^{+}{ }^{1} \mathrm{~S}$ and $\mathrm{Al}^{2} P^{o}$ and corresponding ionization potential IP (in meV).

\begin{tabular}{c|c|c|c|c}
\hline \hline Reference & $E\left(\mathrm{Al}^{+1} S\right)$ & $E\left(\mathrm{Al}^{2} P^{o}\right)$ & $I P$ & $I P(\exp )$. \\
\hline HF & -241.6746681285 & -241.8767049219 & 5497.703 & $5985.768 c$ \\
CISD & -241.7152359492 & -241.9327264044 & 5918.219 & \\
FCI & -241.7152359492 & -241.9352770580 & 5987.626 & \\
FCI $+\Delta E^{\text {OBI }}$ & $-241.715249(1)$ & $-241.935286(1)$ & $5987.51(2)$ & \\
$a$ CVCC & -0.405184154 & -0.405095171 & -2.421 & \\
$b$ rel. & -0.454517980 & -0.454542816 & 0.675 & \\
predicted IP & & & $5985.76(2)$ & \\
\hline
\end{tabular}

$a, b$ - The corresponding energies can be calculated by adding the respective HF energy; $c-[22]$.

TABLE VI

Total energies $E$ (in Ha) on different levels of valence calculations, core-valence and core-core (CVCC) and relativistic effect (rel) for $\mathrm{Al}^{-2} P^{o}$ and $\mathrm{Al}^{-3} P$ and corresponding electron affinity $\mathrm{EA}$ (in meV).

\begin{tabular}{c|c|c|c|c}
\hline \hline Reference & $E\left(\mathrm{Al}^{+1} S\right)$ & $E\left(\mathrm{Al}^{2} P^{o}\right)$ & $I P$ & $I P($ exp. $)$ \\
\hline HF & -241.8767049219 & -241.8782311393 & 41.530 & $432.83(5)$ \\
SD & -241.9327264044 & -241.9460765701 & 363.276 & \\
FCI & -241.9352770580 & -241.9520605599 & 456.702 & \\
FCI $+\Delta E^{\text {OBI }}$ & $-241.935286(1)$ & $-241.952080(1)$ & $456.98(2)$ & \\
$a$ CVCC & -0.405095171 & -0.404543789 & -15.00 & \\
$b$ rel. & -0.454542816 & -0.454205563 & 9.177 & \\
predicted EA & & & $432.81(2)$ & \\
\hline
\end{tabular}

$a, b$ - The corresponding energies can be calculated by adding the respective HF energy.

\section{Conclusion}

We have investigated different contributions to the ionization potential and electron affinity (valence correlation, valence-core and core--core correlation and relativistic effect) of the ground state of aluminum in the frame work of CI. Our calculation includes development of the Slater type orbitals basis set suitable for core-core and core-valence correlation calculations. Our work presents the best result up to date for the ground state of ionization potential and electron affinity of aluminum where FCI truncation energy errors for valence calculation do not exceed $-0.003 \mu \mathrm{Ha}$ for $\mathrm{Al}{ }^{3} P$ state and approximately zero truncation energy error for $\mathrm{Al}^{2} P$.

\section{Acknowledgments}

I am indebted to TWAS-CONACYT fellowship that enabled me to pursue postdoctoral studies at Instituto de Física of Universidad Nacional Autónoma de México. I am also indebted to my supervisor, Professor Carlos F. Bunge, for his continued help, encouragement, criticism and his hospitality during my stay in Mexico.
TABLE VII

A comparison of previous theoretical and present results of ionization potential IP and electron affinity EA. IP and $\mathrm{EA}$ in $(\mathrm{meV})$.

\begin{tabular}{c|c|c|c}
\hline \hline Ref. & Method & IP & EA \\
\hline Arnau et al. [1] & CI-SP & & 450 \\
Woon and Dunning [4] & MRSD-CI & & 437 \\
Eliav et al. [5] & RCC & 5991 & 427 \\
Wijesundera [6] & MCDF & & 433 \\
de Oliveira et al. [7] & CCSD(T) & & 432.77 \\
present work & CI-SDT(Q) & & 432.811 \\
present work & CI-SD(T) & 5985.764 &
\end{tabular}

\section{References}

[1] F. Arnau, F. Mota, J.J. Novoa, J. Chem. Phys. 166, 77 (1992).

[2] T.H. Dunning Jr., J. Chem. Phys. 90, 1007 (1989).

[3] D.E. Woon, T.H. Dunning Jr., J. Chem. Phys. 98, 1358 (1993) 
[4] D.E. Woon, T.H. Dunning Jr., J. Chem. Phys. 99, 3730 (1993).

[5] E. Eliav, Y. Ishikawa, P. Pyykko, U. Kaldor, Phys. Rev. A 56, 4532 (1997).

[6] W.P. Wijesundera, Phys. Rev. A 55, 1785 (1997).

[7] G. de Oliveira, J.M.L. Martin, F. de Proft, P. Geerlings, Phys. Rev. A 60, 1034 (1999).

[8] M. Scheer, R.C. Bilodeau, J. Thogersen, H.K. Haugen, Phys. Rev. A 57, R1496 (1998).

[9] D.E. Woon, T.H. Dunning Jr., J. Chem. Phys. 103, 4572 (1995).

[10] C.F. Bunge, J. Chem. Phys. 125, 014107 (2006).

[11] C.F. Bunge, R. Carbó-Dorca, J. Chem. Phys. 125, 014108 (2006).

[12] P.O. Lowdin, Adv. Chem. Phys. 2, 207 (1959).

[13] C.F. Bunge, Theor. Chim. Acta 16, 126 (1970).
[14] A.V. Bunge, C.F. Bunge, R. Ja'uregui, G. Cisneros, Comp. Chem. 13, 201 (1989).

[15] C.F. Bunge, J.A. Barrientos, A.V. Bunge, At. Data Nucl. Data Tables 53, 113 (1993).

[16] R.E. Brown, Ph.D. Thesis, Department of Chemistry, Indiana University, 1967.

[17] C.F. Bunge, Mol. Phys. 108, 3279 (2010).

[18] C.F. Bunge, Theor. Chim. Acta 126, 139 (2010).

[19] C.X. Almora-Díaz, J. Chem. Phys. 140, 184302 (2014).

[20] C. Schwartz, Phys. Rev. 126, 1015 (1962).

[21] R. Ja'uregui, C.F. Bunge, E. Ley-Koo, Phys. Rev. A 55, 1781 (1997).

[22] NIST Atomic Spectra Database, http://physics.nist.gov/cgi-bin/ASD/ie.pl. 